\title{
3D transient numerical simulation for sabot separation
}

\author{
D. Touati \& S. Peles \\ Israel Military Industries, Central Laboratory Division, P.O.B. 1044, \\ Ramat Hasharon, 47100, Israel
}

\begin{abstract}
This paper presents a sabot discard model to predict sabots separation. The model uses the FSI (Fluid Structure Interaction) numerical packages of MSC-DYTRAN to determine the evolution of sabot separation motion. The 3D model enables us to calculate the asymmetric sabots discard trajectory using Euler Lagrange coupling. We neglect the blast region effect on the sabot discards process near the muzzle. Physical quantities such as linear and angular displacement, velocities, acceleration and exerted forces on the sabot structures are computed. Simulated results and experimental data show close agreement. Keywords: sabots petals, discard, kinetic energy penetrator.
\end{abstract}

\section{Introduction}

The kinetic energy penetrator is a widely used anti tank munitions. Its lethality is due to the kinetic energy imparted by the penetrator to the target with impact velocities of between 1.4 to $1.8 \mathrm{~km} / \mathrm{s}$. The penetrator is launched by means of a sabot assembly, consisting of three aluminum sabot petals, required to minimize the penetrator's in bore balloting. The process of sabot separation begins as the projectile leaves the gun tube. Because of the transverse motion of the projectile within the gun, energy is stored in the elastic sabot petals. As the projectile leaves the muzzle, the constraints of the gun tube are released and the sabot elements are able to move laterally outward. Aerodynamic forces acting on each of the sabot petals causes them to lift up and disengage from the buttress grooves of the penetrator rod to permit its unconstrained, low drag flight to the target. It has been demonstrated by Schmidt and Shear [1] that aerodynamic interference generated by the sabot components can be a significant source of projectile 
launch disturbance leading to unacceptable loss of accuracy at the target. Perturbations to the projectile trajectory are magnified by geometric asymmetry in the discard pattern and by the extended periods during launch when the sabot components are in close proximity to the projectile. Erengil [2] used analytical expressions to estimate transient sabot surface pressure distributions, which are then integrated numerically to yield force and moments to determine sabot discard trajectories. Cayzac et al [3] investigated the launch dynamics of a 44 $\mathrm{mm}$ APFSDS. The 2D Euler equations have been used to predict sabot separation in an intermediate ballistic flow field. Nusca [4] simulated a quasi steady sabot discard process that was also tested in a wind tunnel. First he computes the aerodynamic forces and moments exerted on the sabot petals at specific positions, and then this loading was used to compute the dynamic motion of the sabots. The quasi steady simulation ignores the flow time dynamics and does not link the aerodynamic forces to sabot motion.

An insight into the transient sabots separation processes was provided in this paper via a detailed finite element model, representing the sabot and penetrator subjected to a free stream flow field. The model was prepared using exact geometric part features, which include the buttress grooves details on both the sabot and penetrator.

We used the FSI numerical feature of MSC - DYTRAN, and utilize an Euler Lagrange coupling to correlate unsteady aerodynamic forces and the sabot body motion.

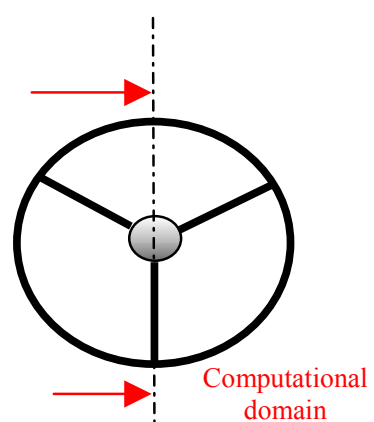

(a)

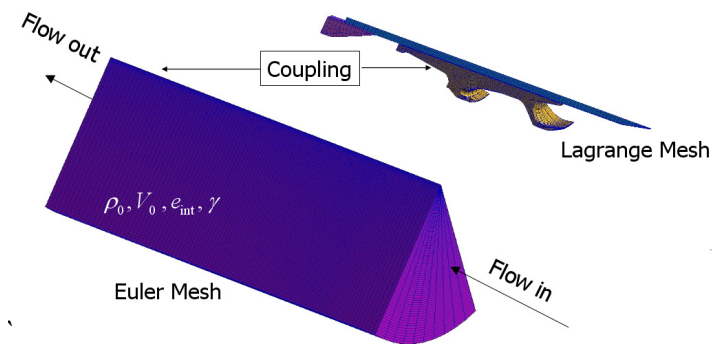

(b)

Figure 1: Slice of the finite elements model of the sabot and projectile rod subjected to free stream flow field.

\section{Numerical model}

The configured numerical model included a stationary fins stabilized penetrator at a zero angle of attack with respect to the free stream flow field, and three $120^{\circ}$ sector sabots petals encompassing the penetrator. The sabot discard simulations are performed for a radial separation of the sabot from the projectile surface. Based on the above assumption, the model utilizes only one half of the computational domain as shown in figure 1a. The flow field characteristics were 
calculated using the Euler equation and the angle of attack was used as the only source of asymmetry in the free flow stream initial condition. A slice of the finite elements model of sabot and penetrator, respectively, subjected to the free stream flow field is shown in Figure 1b.

The numerical model neglected the blast region effect on the sabot discards process near the muzzle based upon experimental results which revealed a short range spatial influence of the blast region on the sabot discard process. The discard process comes to an end at a distance of $15 \mathrm{~m}$ from the muzzle exit while the blast region influence is limited to the close proximity of the muzzle at a distance of $1 \mathrm{~m}$. The sabots and the kinetic projectile rod were modeled using 3 - Dimensional 8 - node Hexagonal elements. The sabot and the penetrator were assumed to be rigid.

The interface at the buttress grooves between the sabot and the penetrator is modeled using contact features of the software to allow for realistic load transfer from the sabot to the penetrator. The interface between the penetrator and sabot buttress groove is shown in enlarged view in figure 2 .

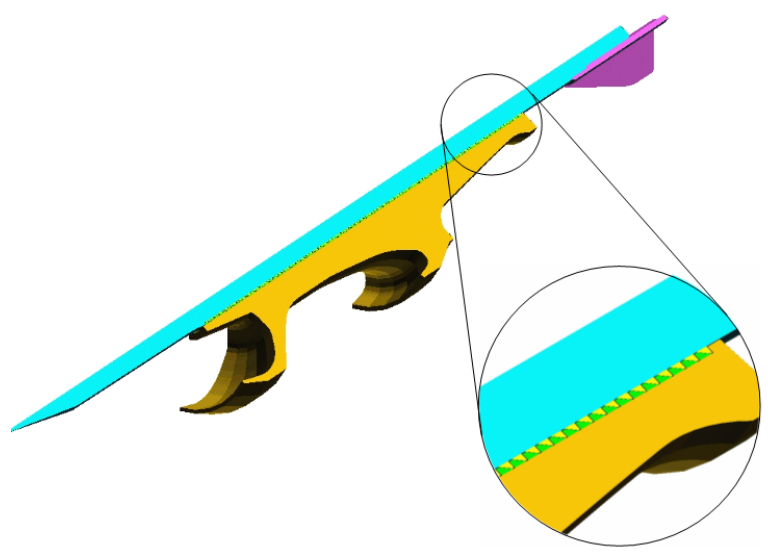

Figure 2: Enlarged view of the sabot and projectile kinetic rod buttress groove interface.

The air surrounding the sabot and main rod structures is modeled using a Eulerian mesh with cylindrical outer shape border. Air inflow and outflow boundary conditions were applied and the air velocity at the boundary was the projectile muzzle velocity which was kept constant throughout the sabot discard simulation. An ideal gas equation of state was used to simulate the air behavior. The dynamic air pressure interacted with the sabots structure by means of a fluid - structure interacting algorithms.

\section{Simulation results}

Figure 3 shows the pressure contours of the air within close proximity of one of the sabot petals. 


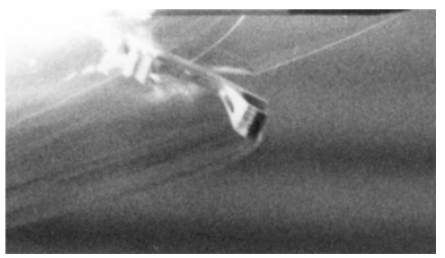

(a)

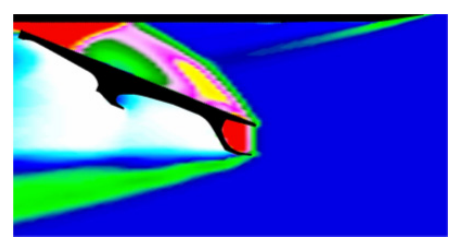

(b)

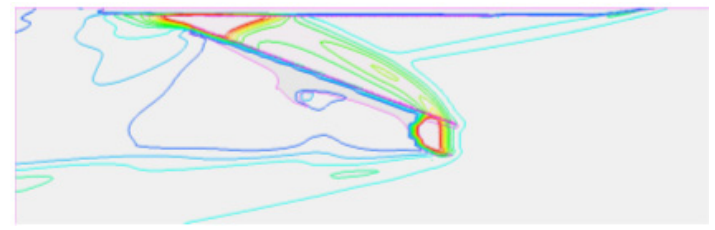

(c)

Figure 3: (a) Shadowgraph photo of sabot discard experiment $3.5 \mathrm{~ms}$ after the projectile exits from bore muzzle. (b) Simulation results of air density plot in close proximity to the sabot region at $3.5 \mathrm{~ms}$. (c) Contour map of density plots (simulation results).
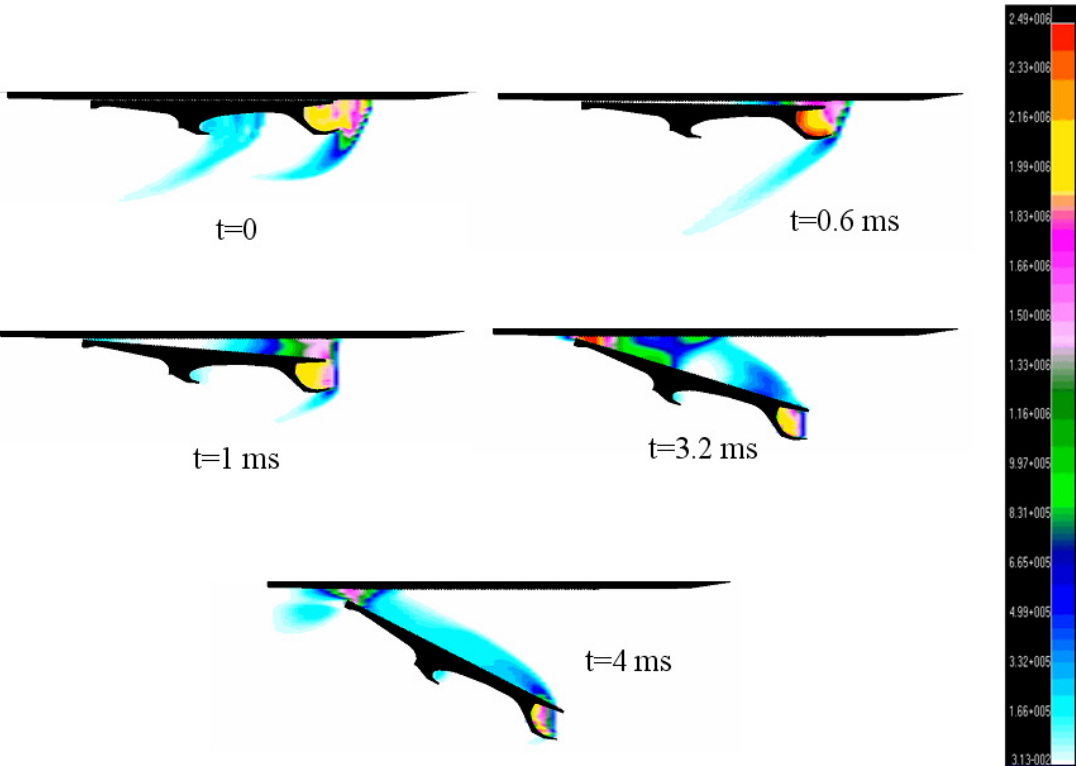

Figure 4: Contours of air pressure surrounding the sabot structure. 
The figure presents the sabot's simulated and experimental attitudes with respect to the projectile rod at $6 \mathrm{~m}$ beyond the muzzle exit. There is a close resemblance between the simulated and experimental shock wave patterns.

Figure 4 presents air pressure contours surrounding the sabot structure at different time frames during the discard process.

Figure 5 depicts the linear velocities components of the sabot's c.g during the discard process at $\left(0^{0}\right.$ angle of attack).

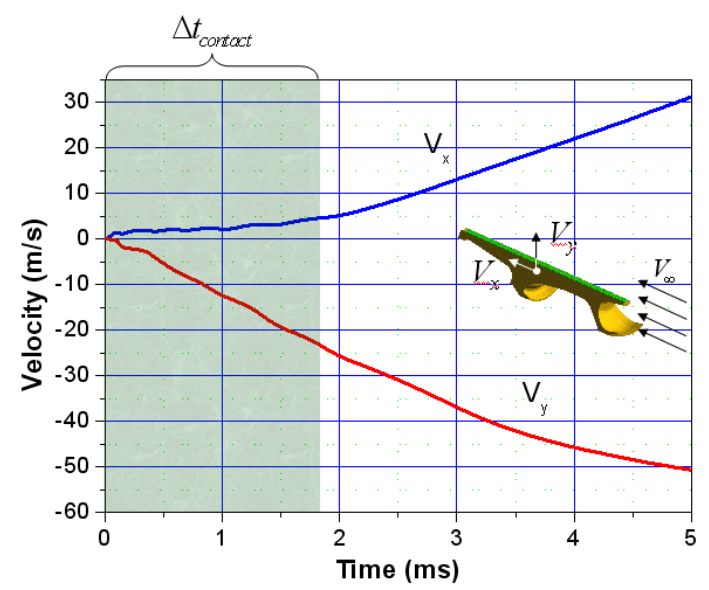

Figure 5: $\quad$ Sabot C.G velocities components $\mathrm{V}_{\mathrm{x}}$ (blue) and $\mathrm{V}_{\mathrm{y}}$ (red).

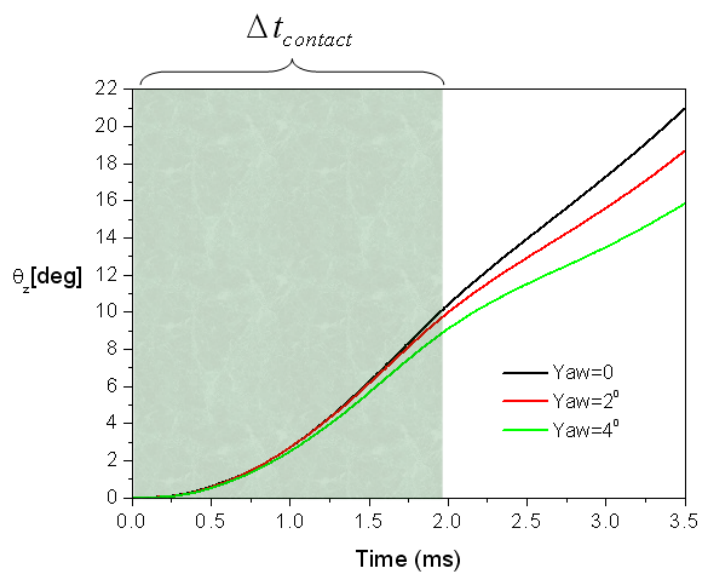

Figure 6: Rotation angle vs. time of upper sabot at three different yaw angles.

Figure 6 shows the sabot's angular displacement during the sabot discard process for different Yaw angles of the free flow. The measured rotation angle around the sabot's c.g. point decreases as the Yaw angle increase. The angular 
differences are due to Yaw angle effects which increase or decrease the petal area which is exposed to the flow field, causing the aerodynamic lift and drag force to discard the sabot at a slower or faster rate correspondingly. As may be seen, the interaction time between the sabot and the kinetic projectile rod does not exceed $2 \mathrm{~ms}$ for the specific sabot geometry and the given yaw angles.

Figure 7 shows the aerodynamics force components exerted on the sabot during the discard process. The sabot separation process involves a continuous growth of the sabot surface area exposed to the free stream flow which results in an increase in the corresponding aerodynamic force magnitude in the $\mathrm{x}$ direction from $1 \mathrm{KN}$ up to almost $10 \mathrm{KN}$ while the resultant aerodynamic force magnitude in the $\mathrm{y}$ direction decreases.

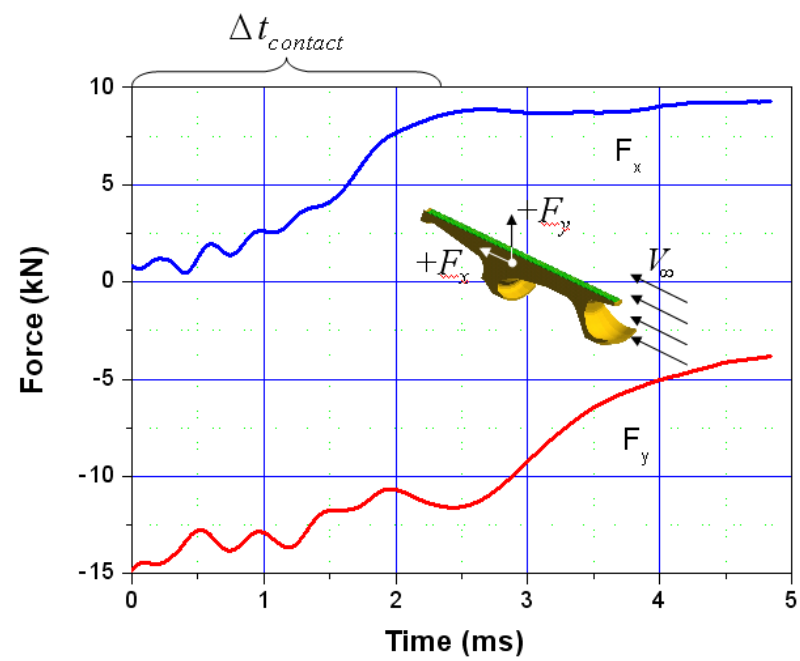

Figure 7: Aerodynamic forces exerted on sabot structure.

\section{Summary}

An insight into the transient sabots separation processes was provided in this paper via a detailed finite element model, representing the sabot and penetrator, subjected to a free stream flow field. The model was prepared using exact geometric part features, which include the buttress groove details on both the sabot and penetrator.

It was written in the FSI numerical package of MSC - DYTRAN and utilizes an Euler Lagrange coupling to correlate the unsteady aerodynamic forces and the sabots' rigid body motions. In retrospect we determined that the fluid Euler equation are sufficient for describing the sabot's entire spatial motion and the general characteristics of the flow field over the sabot. The magnitude of the exerted aerodynamic interfacial forces were assessed, and it was further revealed that the interaction time between the sabot and the projectile rod does not exceed 
$2 \mathrm{~ms}$ for the specific sabot geometry. The most significant attribute of this model is that it does not rely on any empirical data and is therefore widely applicable. The simulation results are in close agreement with the experiments.

\section{References}

[1] Schmidt E.M., Shear D.D., Aerodynamic Interface During Sabot Discard, Journal of Spacecraft and Rockets, AIAA, Vol 15, No 3, May-June, 1978, pp. 235-240.

[2] Erengil M. E., Sabot Discard Model For Conventional and Electromagnetic Launch Package, $19^{\text {th }}$ International Symposium of Ballistics 7-11 May 2001, pp 213-221 Interlaken, Switzerland.

[3] Cayzac R., Carette E., Alziary de Roquefort T. Intermediate Ballistics Unsteady Sabot Separation: First Computations and Validation, $19^{\text {th }}$ International Symposium of Ballistics 7-11 May 2001, pp 297-305 Interlaken, Switzerland.

[4] Nusca M. J., Numerical Simulation of Sabot Discard Aerodynamics, Army Research Laboratory ARL-TR-204 September 1993. 